\title{
Management of transient ischemic attack or nondisabling stroke related to extracranial internal carotid artery stenosis
}

\author{
Varun Kapila MD, Prasad Jetty MD MSc, Vincenzo S. Basile MD BEd, Luc Dubois MD MSc
}

Cite as: CMAJ 2019 April 15;191:E418-22. doi: 10.1503/cmaj.180735

CMAJ Podcasts: author interview at https://soundcloud.com/cmajpodcasts/180735-view

erebrovascular disease is not only the third largest cause of death in Canada, it is also associated with substantial functional morbidity after an event. In 2013, over 400000 Canadians were living with the disabling effects of a stroke, most of which were due to ischemic injury. ${ }^{1}$ Extracranial internal carotid artery embolic events account for about $10 \%-$ $15 \%$ of all strokes. ${ }^{2}$ Symptoms usually occur because of atherosclerotic plaque rupture in the internal carotid artery and embolization of plaque particles to the brain, which leads to transient or persistent ischemia in a specific anatomic area and related neurologic symptoms.

Population-based studies have suggested a high risk of recurrent transient ischemic attack (TIA) or stroke after an index event from a moderate to severe stenosis of the extracranial internal carotid artery, ranging from $10 \%$ to $20 \%$ within the next 90 days. ${ }^{3}$ Subsequently, a systematic review and meta-analysis found that most of these events occurred within the first 48 hours to 7 days, ${ }^{4}$ which suggests that risk is front loaded, and, consequently, an imperative to identify urgently and treat these patients at high risk to avoid the devastating consequences of a future stroke

\section{KEY POINTS}

- The diagnosis of symptomatic events from a moderate to severe extracranial carotid artery stenosis $(50 \%-99 \%)$ is a medical emergency.

- Patients should be admitted, with urgent revascularization within the next few days when possible.

- Evidence supports use of dual-antiplatelet therapy initiated early, limited in duration ( $21 \mathrm{~d}$ ) and then downgraded to monotherapy after intervention to maximize the early secondary prevention benefit and reduce bleeding risks.

- The resolution of symptoms should not provide false reassurance; risks of recurrent events remain high without revascularization.

event. Management of symptomatic extracranial internal carotid artery stenosis, both medical and surgical, is aimed at reducing the likelihood that further plaque debris will embolize to the brain (Figure 1).

We review the medical and surgical management of patients with symptomatic extracranial internal carotid artery stenosis,
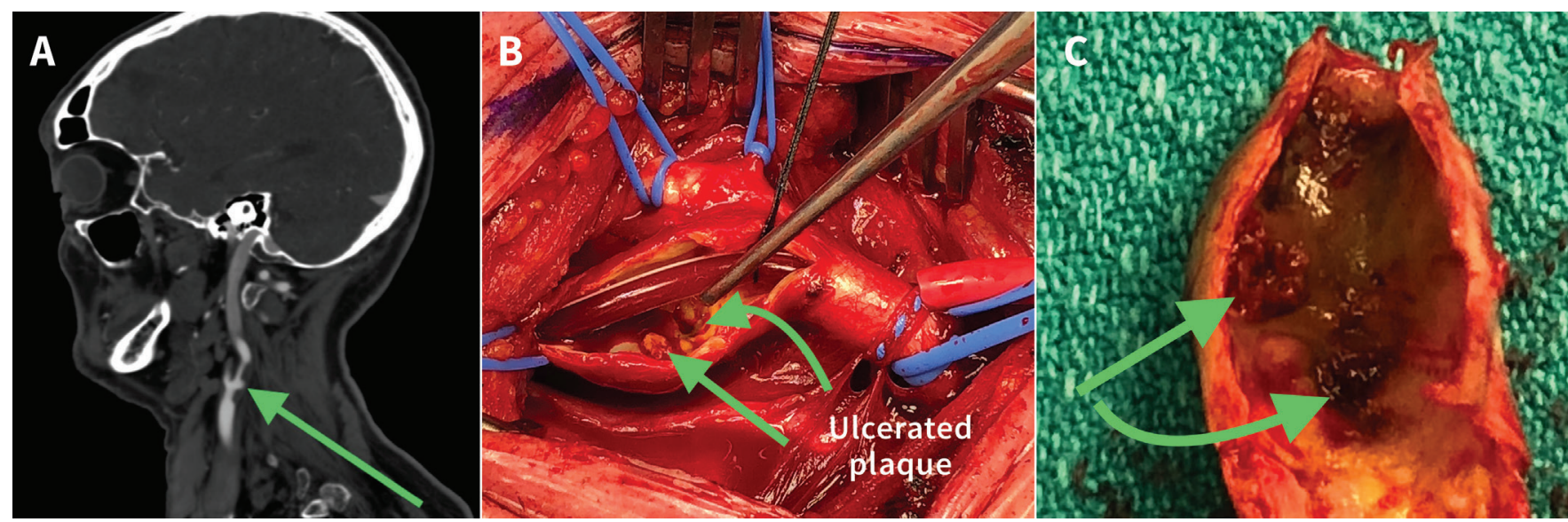

Figure 1: (A) Sagittal slice of a computed tomography angiogram, (B) intraoperative view showing the open and shunted internal carotid artery, and (C) the endarterectomized specimen that was removed from a patient who underwent emergent carotid artery endarterectomy for a nondisabling stroke. The images show the irregular, embolic-producing and ulcerative nature (green arrows) of a typical plaque in an acutely symptomatic patient. 


\section{Box 1: Evidence used in this review}

Given the large number of carotid-related publications, we conducted 4 question-specific narrative searches of PubMed that reflected our 4 main questions. There were no language or time restrictions. We focused our conclusions on randomized controlled trials, large observational cohorts, Canadian data (where available) and meta-analyses. A total of 88 articles were identified. We added additional articles for review. The search strategy for the literature review is listed in Appendix 1.

focusing on recent guidelines, large trials and meta-analyses, and highlighting recent evidence supporting early carotid intervention (Box 1; Appendix 1, available at www.cmaj.ca/lookup/ suppl/doi:10.1503/cmaj.180735/-/DC1).

\section{What are the clinical features of a transient ischemic attack or minor stroke related to internal carotid artery stenosis?}

Symptoms and signs of a localized neurologic deficit are the hallmark of a TIA or minor stroke event. Global or bilateral symptoms and those that are not localized to a specific part of the brain are unlikely to be associated with an extracranial internal carotid artery plaque rupture (Box 2). It is outside the scope of this article to discuss these other diagnoses in detail.

\section{What medical treatment should be started for acute transient ischemic attack or nondisabling strokes?}

The current aim of medical treatment for the management of symptomatic extracranial internal carotid artery stenosis is to reduce the likelihood of further embolic events originating from the internal carotid artery plaque. Medical management aims to prevent further events until the carotid lesion can be treated and, secondarily, to manage risk factors that are common to cardiac atherosclerotic disease and thereby reduce the risk of future cardiac ischemic events. The 2 mainstays of acute management are antiplatelet agents and statin therapy. Long-term risk factor management - not discussed here - includes assistance with smoking cessation, diet and lifestyle educational interventions, glucose and cholesterol control, and management of hypertension.

\section{Antiplatelet treatment}

Treatment options include single antiplatelet versus dual antiplatelet regimens, unfractionated heparin or full anticoagulation, or a combination of modalities. Monotherapy with a single antiplatelet agent has been the mainstay of treatment for years. A 2016 meta-analysis showed that early initiation of acetylsalicylic acid (ASA) alone after a TIA or ischemic stroke reduced subsequent events by $60 \% .^{5}$

However, 2 recent large randomized controlled trials (RCTs) investigated dual-antiplatelet treatment in patients with acute
Box 2: Signs or symptoms possibly or unlikely to be related to an extracranial internal carotid artery embolic event*

\begin{tabular}{ll}
$\begin{array}{l}\text { Signs or symptoms } \\
\text { suggestive of an extracranial } \\
\text { ICA event }\end{array}$ & $\begin{array}{c}\text { Signs or symptoms unlikely } \\
\text { to be related to an extracranial } \\
\text { ICA event }\end{array}$ \\
$\begin{array}{ll}\text { - Unilateral extremity motor } \\
\text { or sensory impairment } \\
\text { (transient, fluctuating or }\end{array}$ & - Dizziness \\
persistent) & - Vertigo \\
- Monocular vision loss & Bilateral visual disturbances \\
$\begin{array}{ll}\text { - Acute speech deficit or } \\
\text { change }\end{array}$ & - Headache \\
\hline Facial droop & Bilateral extremity motor or \\
& sensory impairment \\
\hline Note: ICA = internal carotid artery. & Ataxia \\
\hline *Symptoms of a carotid event are related to the specific area of the brain that is \\
affected, resulting in a focal neurologic deficit.
\end{tabular}

symptoms of ischemic stroke. The investigators from the Clopidogel in High-Risk Patients with Acute Nondisabling Cerebrovascular Events (CHANCE) trial compared the benefit of dual antiplatelet therapy over ASA alone in the acute management of over 5000 patients in China with nondisabling stroke or high-risk TIA. ${ }^{6}$ A dual antiplatelet regimen of clopidogrel, administered with a $300 \mathrm{mg}$ initial dose followed by $75 \mathrm{mg}$ daily for 90 days, added to ASA (75 mg daily) given over the first 21 days, reduced the likelihood of stroke events over 90 days compared with ASA alone (hazard ratio $0.68,95 \%$ [confidence interval] $\mathrm{Cl} 0.57-0.81$ ). No increased bleeding events were noted in the group receiving dual-antiplatelet treatment. However, these findings cannot be generalized beyond the Chinese population.

More recently, the Platelet-Oriented Inhibition in New TIA and Minor Ischemic Stroke (POINT) trial investigators compared a $600 \mathrm{mg}$ loading dose of clopidogrel followed by $75 \mathrm{mg}$ per day with ASA (50-325 mg/d) versus ASA alone in an international population of 4881 patients. ${ }^{7}$ The duration of treatment studied in the POINT trial exceeded that in the CHANCE trial, and the investigators looked at ischemic events after 90 days of treatment rather than 21 days. Although there was a significant reduction in major ischemic events at 90 days in the dual-antiplatelet arm ( $5 \%$ v. $6.5 \%)$, the POINT trial was stopped early because of increased major hemorrhage occurring in patients in the dualantiplatelet arm (0.9\% v. $0.4 \%)$. Most of the bleeding events were nonintracranial, with no difference in death from such events in either arm.

An important limitation of the POINT trial was that patients who underwent a carotid intervention were excluded. The risks of surgery among patients on dual antiplatelet therapy must be considered; some surgeons have concerns about the potential for neck hematoma owing to bleeding after carotid artery endarterectomy. This is especially true, given that the POINT trial excluded these patients, which may mitigate some of the benefits seen in this study. 
The findings from the CHANCE and the POINT trials suggest that dual-antiplatelet therapy may reduce the risk of further neurologic events in symptomatic patients with a minor stroke or high-risk TIA more than treatment with ASA alone. Differences between the trials in observed bleeding events may be attributed to the longer duration of dual-antiplatelet therapy in the POINT trial (90 d v. $21 \mathrm{~d}$ ). The intensity of the loading dose used by the POINT trial investigators could be a concern; however, the increased bleeding risk occurred between 8 and 90 days and not during the first 7 days in the POINT trial.

Because dual-antiplatelet therapy in the acute period after a nondisabling stroke or TIA has been shown to reduce rates of recurrent stroke, this approach should be considered at least for 21 days. Use of short-term, early dual-antiplatelet therapy is supported by the 2018 Canadian Stroke Best Practice Recommendations for the Secondary Prevention of Stroke, ${ }^{8}$ the 2018 Guidelines for the Early Management of Patients with Acute Ischemic Stroke from the American Heart Association/American Stroke Association ${ }^{9}$ and the 2017 European carotid guidelines ${ }^{10}$ as Level $\mathrm{A}, \mathrm{B}$ and $\mathrm{C}$ recommendations, respectively.

\section{Statin therapy}

The long-term benefit of statins for patients at risk for atherosclerotic events is well-established, including after an ischemic stroke event. Since 2002, after the publication of findings from the Medical Research Council (MRC)/British Heart Foundation (BHF) Heart Protection Study, ${ }^{11}$ statins have been used to decrease mortality in patients at risk for cardiovascular, peripheral arterial or stroke events. The pleiotropic effect of statins is well documented; they appear to confer benefits beyond their lipid-lowering effect. A 2012 meta-analysis of individual patient data from 27 RCTs found a significant dose-dependant benefit of statin use in patients at risk for vascular events. ${ }^{12}$ Every $1 \mathrm{mmol} / \mathrm{L}$ reduction in low-density lipoprotein (LDL) was associated with about a $25 \%$ reduction in cardiovascular mortality, and an overall $15 \%$ reduction in risk of stroke events. The Stroke Prevention by Aggressive Reduction in Choleterol Levels (SPARCL) trial investigators evaluated the effect of an aggressive lipid-lowering regimen of high-dose atorvastatin ( $80 \mathrm{mg}$ daily) in patients 1-6 months after a stroke or TIA and found a relative risk reduction of $33 \%$ in fatal and nonfatal strokes over almost 5 years. ${ }^{13}$

There are, however, limited data on the effectiveness of statin treatment immediately after TIA or nondisabling strokes. A retrospective review of symptomatic patients who underwent carotid artery endarterectomy in Western Canada found that use of a statin reduced in-hospital death by $75 \%$ and ischemic stroke or death by $45 \%$ (adjusted odds ratios $0.25,95 \% \mathrm{Cl} 0.07-0.90$, and $0.55,95 \% \mathrm{Cl} 0.32-0.95$, respectively). ${ }^{14} \mathrm{~A} 2005$ retrospective study estimated that use of preoperative statins reduced rates of perioperative stroke by $73 \%$ compared with no statin use $(1.3 \% \mathrm{v}$. 4.5\%). ${ }^{15}$ However, no evidence from RCTs is available to support the idea that immediate statin initiation after TIA or nondisabling symptomatic stroke improves early outcomes.

Recent Canadian, American and European guidelines recommend starting treatment with a statin for long-term prevention of stroke, myocardial infarction (MI) and other vascular events in patients with recently symptomatic ischemic neurologic events. ${ }^{8-10}$ Currently, in contrast to the recommendations for starting antiplatelet therapy, there are no Level A recommendations (i.e., based on evidence from RCTs) for immediate or intensive initiation of statin therapy for reducing early stroke events.

\section{When should carotid intervention occur?}

"Carotid intervention" includes carotid artery endarterectomy and carotid artery stenting. Decades ago, intervention for secondary prevention after an embolic extracranial stroke of internal carotid artery origin was delayed for 4-8 weeks to allow the brain to "recover." This was done in the hopes of avoiding consequences of early reperfusion such as cerebral edema and hemorrhage. However, excessive delay to intervention resulted in a prohibitively large number of recurrent stroke events. ${ }^{16}$

A 2004 pooled analysis of data from 2 large RCTs showed a clear benefit for carotid artery endarterectomy in patients with recent symptoms from extracranial internal carotid artery stenoses. ${ }^{17}$ The number needed to treat was 6 for patients with a severe (70\%-99\%) ipsilateral carotid stenosis and 13 for those with moderate (50\%-69\%) stenosis.

\section{Emergent treatment}

The benefit of carotid intervention has been shown to be based on several factors, including degree of stenosis, patient sex and time to intervention, with earlier intervention - within 2 weeks - being associated with a greater secondary preventative benefit, and a longer delay associated with less benefit to the patient. ${ }^{16}$ This evidence underpinned previous recommendations by national guidelines that advocated intervention within 14 days. ${ }^{10,18}$

However, more recent evidence has shown a high risk of recurrent events within the first few days to weeks after a TIA or minor stroke without intervention. ${ }^{17}$ A 2015 systematic review estimated the risks of a repeat neurologic event to be $6 \%$ within 2-3 days and as high as $19.5 \%$ within 7 days of TIA or minor stroke related to stenosis of the extracranial internal carotid artery. ${ }^{19}$ This has led to a shift to more urgent scheduling of carotid interventions in patients with internal carotid artery-related stroke or TIA and a recent change to the Canadian guideline.

The 2018 Canadian Stroke Best Practice Recommendations now state that patients with a TIA or nondisabling stroke, who present with "transient, fluctuating or persistent unilateral motor weakness or speech disturbance/aphasia or ocular symptoms," should undergo carotid intervention as soon as possible if they have moderate to severe stenosis of the extracranial internal carotid artery (50\%-99\%), ${ }^{8}$ ideally within the first few days of presentation when reasonable. Such patients should not be discharged home but rather be admitted for emergent work-up, medical treatment and expedited intervention in an effort to prevent another - potentially disabling - embolic stroke event. Patients with a TIA or minor stroke and severe extracranial internal carotid artery stenosis should be treated with similar urgency to patients with an MI or unstable angina. 


\section{Controversy}

Many studies that assessed carotid artery endarterectomy performed within 48 hours of symptom onset found acceptable risks of perioperative stroke and death, ${ }^{20-23}$ whereas the Carotid Alarm Study found higher risk of complications if intervention was performed within 48 hours. ${ }^{24}$ A 2018 meta-analysis of RCTs and observational studies that compared stroke rates between very urgent (< $48 \mathrm{~h}$ from symptom onset) and urgent endarterectomy ( $>48 \mathrm{~h}$ ) found higher stroke rates after very urgent endarterectomy. ${ }^{25}$

Although there is no RCT evidence to guide timing of early carotid intervention, there may be a benefit to waiting beyond 48 hours after symptom onset to minimize the risk of postoperative stroke; however, beyond that, surgery should occur as soon as possible and ideally within the first few days postevent. The 2018 American stroke guideline recommends that carotid intervention occur between 48 hours and 7 days of symptom onset. ${ }^{9}$

Regardless of the timing to intervention, the timing to first assessment by a specialist in carotid intervention should be considered urgent, to hasten creation of a patient-specific treatment plan aimed at reducing further embolic events.

\section{Special considerations}

There are 2 specific subgroups of patients that arguably should not receive carotid intervention within the guideline-recommended time period. At 1 end of the spectrum, patients with crescendo TIAs have a devastatingly high risk of another stroke event early on and should undergo carotid intervention within 24 hours. ${ }^{10}$ At the other end of spectrum certain patients, including those with a large territory of ischemic brain ( $>1 / 3$ of the middle cerebral artery territory) or evidence of ischemic hemorrhage, may benefit from purposeful delay to carotid intervention (> 2-4 wk) because they are at elevated risk of postintervention cerebral reperfusion, hemorrhage and subsequent complications, and these should be weighed against the risk of recurrent stroke. ${ }^{10}$ Both of these recommendations are supported in the 2017 European guideline ${ }^{10}$ but are not specifically addressed in the 2018 Canadian or American guidelines. ${ }^{8,9}$

\section{Should patients undergo carotid artery endarterectomy or stenting?}

The large Carotid Revascularization Endarterectomy vs. Stenting Trial (CREST), which compared carotid artery endarterectomy to carotid artery stenting, found that the aggregate outcome of stroke, MI or death from any cause during the periprocedural period or any ipsilateral stroke within 4 years after random assignment was similar for both groups. ${ }^{26}$ However, if the goal of intervention is stroke prevention, then the compound end point is somewhat misleading. When assessed for stroke outcomes, patients who received carotid artery stenting had double the risk of periprocedural stroke or death compared with those who received carotid artery endarterectomy (4.1\% v. $2.3 \%) .{ }^{26}$ In the long term, the risks of ipsilateral stroke were similar in both groups. However, carotid artery stenting did show a reduced rate of periprocedural MI compared with endarterectomy (1.1\% v. $2.3 \%)$ and fewer local complications. In a 2018 comparison of more than 15000 carotid interven- tions performed in Ontario, carotid artery endarterectomy was associated with a 55\% lower risk of adverse events when compared with carotid artery stenting. ${ }^{27}$

Carotid artery stenting may have a role in the management of some patients (e.g., those with unstable cardiac or respiratory conditions, or patients with high-carotid artery bifurcation or long lesions) who are less likely to do well with carotid artery endarterectomy. However, evidence suggests that the best choice of carotid intervention remains carotid artery endarterectomy for all symptomatic patients with moderate to severe carotid artery stenosis. A pooled analysis of the 3 largest trials examining carotid artery endarterectomy versus carotid artery stenting found that the highest relative risk of periprocedural stroke events (stenting v. endarterectomy) occurred in the first 7 days after the procedure, ${ }^{28}$ further supporting the choice of endarterectomy over stenting, since carotid intervention is usually undertaken more urgently now.

\section{Conclusion}

Symptomatic extracranial moderate to severe carotid artery stenosis, if left untreated, can have devastating consequences. Given the high risk of further neurologic events in the period immediately following the initial presentation, urgent admission is warranted to ensure early assessment by a specialist in carotid disease, with a view to urgent carotid intervention - ideally carotid artery endarterectomy for patients with more than $50 \%$ symptomatic extracranial internal carotid artery stenosis. Physicians should not be falsely reassured if the index event was transient and assume that early symptom resolution implies urgent intervention is not required, because the resolution of symptoms does not predict a more favourable profile.

The new Canadian guideline recommends carotid intervention within the first few days after a nondisabling stroke or TIA. Dual antiplatelet therapy is warranted immediately but should be limited to a short period (less than 21 days) because of the risk of bleeding. Statin therapy should be considered for secondary prevention; however, its benefit for reduction in early recurrent events is not clear.

\section{References}

1. Krueger $\mathrm{H}$, Koot J, Hall RE, et al. Prevalence of individuals experiencing the effects of stroke in canada: trends and projections. Stroke 2015;46:2226-31.

2. Ay H, Arsava EM, Andsberg G, et al. Pathogenic ischemic stroke phenotypes in the NINDS-stroke genetics network. Stroke 2014;45:3589-96.

3. Lovett JK, Coull AJ, Rothwell PM. Early risk of recurrence by subtype of ischemic stroke in population-based incidence studies. Neurology 2004;62:569-73.

4. Giles MF, Rothwell PM. Risk of stroke early after transient ischaemic attack: a systematic review and meta-analysis. Lancet Neurol 2007;6:1063-72.

5. Rothwell PM, Algra A, Chen Z, et al. Effects of aspirin on risk and severity of early recurrent stroke after transient ischaemic attack and ischaemic stroke: time-course analysis of randomised trials. Lancet 2016;388:365-75.

6. Wang Y, Johnston SC, Wang Y. Clopidogrel with aspirin in minor stroke or transient ischemic attack. N Engl J Med 2013;369:1376-7.

7. Johnston SC, Easton JD, Farrant M, et al.; Clinical Research Collaboration, Neurological Emergencies Treatment Trials Network, and the POINT Investigators. Clopidogrel and aspirin in acute ischemic stroke and high-risk TIA. N Engl J Med 2018;379:215-25.

8. Wein T, Lindsay MP, Côté R, et al.; Heart and Stroke Foundation Canadian Stroke Best Practice Committees. Canadian stroke best practice recommendations: secondary prevention of stroke, sixth edition practice guidelines, update 2017. Int J Stroke 2018;13:420-43. 
9. Powers WJ, Rabinstein AA, Ackerson T, et al.; American Heart Association Stroke Council. 2018 Guidelines for the early management of patients with acute ischemic stroke: a guideline for healthcare professionals from the American Heart Association/American Stroke Association. Stroke 2018;49:e46-110.

10. Naylor AR, Ricco JB, de Borst GJ, et al. Editor's choice - Management of atherosclerotic carotid and vertebral artery disease: 2017 clinical practice guidelines of the European Society for Vascular Surgery (ESVS). Eur J Vasc Endovasc Surg 2018;55:3-81.

11. Heart Protection Study Collaborative Group. MRC/BHF Heart Protection Study of cholesterol lowering with simvastatin in 20,536 high-risk individuals: a randomised placebo-controlled trial. Lancet 2002;360:7-22.

12. Cholesterol Treatment Trialists' (CTT) Collaborators; Mihaylova B, Emberson J, Blackwell L, et al. The effects of lowering LDL cholesterol with statin therapy in people at low risk of vascular disease: meta-analysis of individual data from 27 randomised trials. Lancet 2012;380:581-90.

13. Amarenco P, Benavente O, Goldstein LB, et al.; Stroke Prevention by Aggressive Reduction in Cholesterol Levels Investigators. Results of the Stroke Prevention by Aggressive Reduction in Cholesterol Levels (SPARCL) trial by stroke subtypes. Stroke 2009;40:1405-9.

14. Kennedy J, Quan H, Buchan AM, et al. Statins are associated with better outcomes after carotid endarterectomy in symptomatic patients. Stroke 2005;36:2072-6.

15. McGirt MJ, Perler BA, Brooke BS, et al. 3-Hydroxy-3-methylglutaryl coenzyme A reductase inhibitors reduce the risk of perioperative stroke and mortality after carotid endarterectomy. J Vasc Surg 2005;42:829-36, discussion 836-7.

16. Naylor AR. Delay may reduce procedural risk, but at what price to the patient? Eur J Vasc Endovasc Surg 2008;35:383-91.

17. Rothwell PM, Eliasziw M, Gutnikov SA, et al.; Carotid Endarterectomy Trialists Collaboration. Endarterectomy for symptomatic carotid stenosis in relation to clinical subgroups and timing of surgery. Lancet 2004;363:915-24.

18. Kernan WN, Ovbiagele B, Black HR, et al.; American Heart Association Stroke Council, Council on Cardiovascular and Stroke Nursing, Council on Clinical Cardiology, and Council on Peripheral Vascular Disease. Guidelines for the prevention of stroke in patients with stroke and transient ischemic attack: a guideline for healthcare professionals from the American Heart Association/ American Stroke Association [published erratum in Stroke 2015;46:e54]. Stroke 2014;45:2160-236.

19. Tsantilas $P$, Kühn A, Kallmayer M, et al. Stroke risk in the early period after carotid related symptoms: a systematic review. J Cardiovasc Surg (Torino) 2015;56: 845-52.

20. Capoccia L, Sbarigia E, Speziale F, et al. Urgent carotid endarterectomy to prevent recurrence and improve neurologic outcome in mild-to-moderate acute neurologic events. J Vasc Surg 2011;53:622-7, discussion 627-8.

21. Ferrero E, Ferri M, Viazzo A, et al. A retrospective study on early carotid endarterectomy within 48 hours after transient ischemic attack and stroke in evolution. Ann Vasc Surg 2014;28:227-38.

22. Tsantilas $P$, Kühnl A, Kallmayer M, et al. A short time interval between the neurologic index event and carotid endarterectomy is not a risk factor for carotid surgery. J Vasc Surg 2017;65:12-20.e1.

23. Rantner B, Schmidauer C, Knoflach M, et al. Very urgent carotid endarterectomy does not increase the procedural risk. Eur J Vasc Endovasc Surg 2015;49:129-36.

24. Nordanstig A, Rosengren L, Strömberg S, et al. Editor's choice - Very urgent carotid endarterectomy is associated with an increased procedural risk: the Carotid Alarm Study. Eur J Vasc Endovasc Surg 2017;54:278-86.

25. Milgrom D, Hajibandeh S, Hajibandeh S, et al. Editor's choice - Systematic review and meta-analysis of very urgent carotid intervention for symptomatic carotid disease. Eur J Vasc Endovasc Surg 2018;56:622-31.

26. Brott TG, Hobson RW II, Howard G, et al.; CREST Investigators. Stenting versus endarterectomy for treatment of carotid-artery stenosis [published errata in N Engl J Med 2010;363:198; N Engl J Med 2010;363:498]. N Engl J Med 2010;363: 11-23.

27. Hussain MA, Mamdani M, Tu JV, et al. Long-term outcomes of carotid endarterectomy versus stenting in a multicenter population-based Canadian study. Ann Surg 2018;268:364-73.

28. Rantner B, Goebel G, Bonati LH, et al.; Carotid Stenting Trialists' Collaboration. The risk of carotid artery stenting compared with carotid endarterectomy is greatest in patients treated within 7 days of symptoms. J Vasc Surg 2013;57: 619-26.e2; discussion 625-6.

\section{Competing interests: None declared.}

This article has been peer reviewed.

Affiliations: Division of Vascular Surgery (Kapila), William Osler Health System, Brampton, Ont.; Division of Vascular Surgery (Jetty), University of Ottawa, Ottawa, Ont.; Division of Neurology, Mackenzie Health System (Basile), Vaughan, Ont.; Division of Vascular Surgery (Dubois), Western University, London, Ont.
Contributors: Varun Kapila conceived the work. Prasad Jetty and Luc Dubois coordinated the drafting of the manuscript and the literature search. All of the authors drafted the manuscript, reviewed the literature, revised the work critically for important intellectual content, gave final approval of the version to be published and agreed to be accountable for all aspects of the work.

Correspondence to: Varun Kapila, varun.kapila@williamoslerhs.ca 